\title{
An 81-Year-Old Woman with Recalcitrant Blisters
}

\author{
Daniel C. Butler \\ Harvard Combined Dermatology Residency Program, and Departments of Pathology and \\ Dermatology, Massachusetts General Hospital, Boston, MA, USA
}

\section{Keywords}

Epidermolysis bullosa acquisita · Blisters $\cdot$ Salt split

\section{Abstract}

Epidermolysis bullosa acquisita (EBA) is a rare mucocutaneous blistering disorder with typical onset in adulthood. Diagnosis and management can be difficult owing to the variability in presentation and clinical manifestation. In this case, we explore a case of EBA as well as provide a general overview of the condition and its variants.

\section{Case Report}

An 81-year-old female with a history of type II diabetes and hepatitis B presented to the Massachusetts General Hospital after a traumatic fall. The patient was found to have a femoral neck fracture; however, the dermatology department was consulted as blisters and erosions were concurrently noted on the dorsal feet, lower legs, and dorsal hands. After evaluation by the dermatology department where biopsies were taken for hematoxylin and eosin staining as well as direct immunofluorescence, the patient was taken to surgery for fracture repair. On the follow-up evaluation, 1 day after the operation, at the site of a dressing change on the left hip, the patient had a large geometric patch of denudation corresponding to the areas where adhesive tape had been removed from the skin (Fig. 1a-c, 2a-e; photo credit: Dr. Cynthia Magro, Weill-Cornell New York Hospital).

\section{Diagnosis and Clinical Course}

Histopathologic evaluation showed a subepidermal blister with a superficial perivascular lymphocytic infiltrate. Eosinophils were not conspicuous. The differential diagnosis

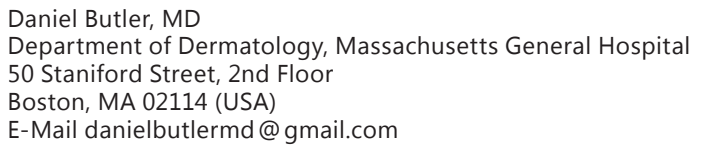




\section{Dermato pathology}

\begin{tabular}{l|l}
\hline Dermatopathology 2018;5:1-5 \\
\hline DOI: 10.1159/000481529 & $\begin{array}{l}\text { @ 2018 The Author(s). Published by S. Karger AG, Basel } \\
\text { www.karger.com/dpa }\end{array}$ \\
\hline
\end{tabular}

Butler et al:: An 81-Year-Old Woman with Recalcitrant Blisters
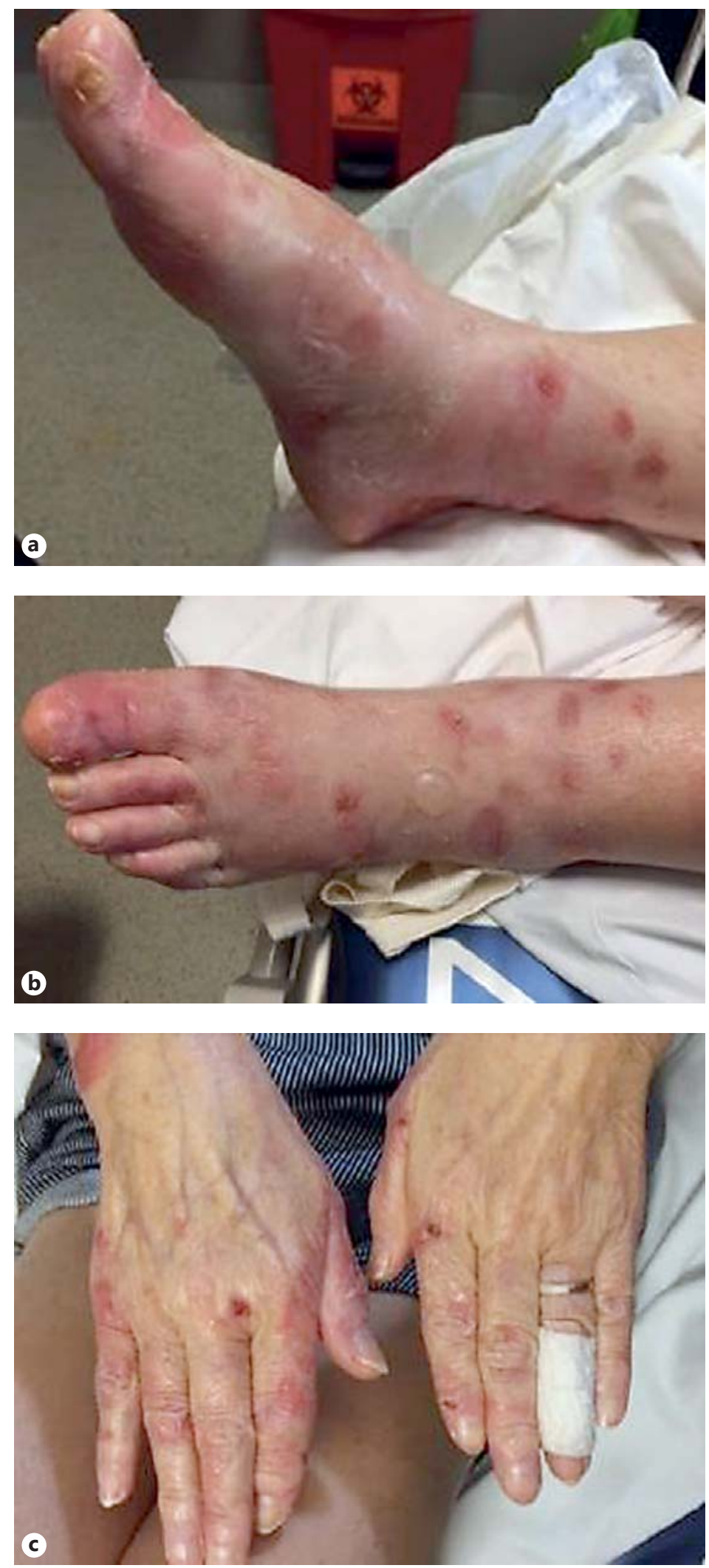

Fig. 1. a-c Distal digits, dorsal feet, and dorsal hands with circular erosions with a macular pink periphery as well as intact bullae. Negative Nikolsky sign. (Of note, the 1 st toe nail was surgically removed in childhood and does not reflect disease activity). 

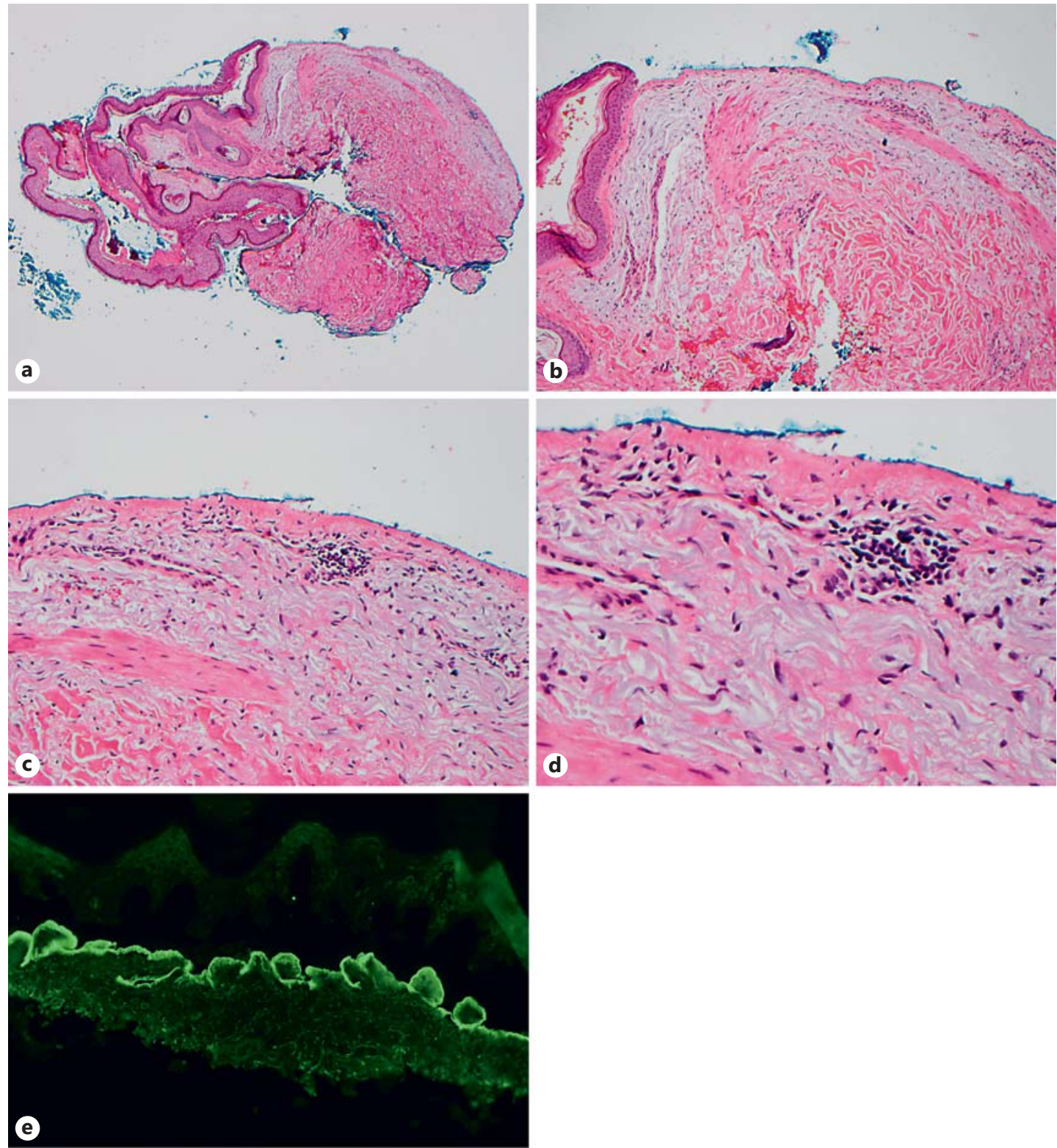

Fig. 2. Pathology images of a bullous lesion. a H\&E. Magnification $\times 10$. b H\&E. Magnification $\times 40$. $\mathbf{c} H \& E$. Magnification $\times 100$. d H\&E. Magnification $\times 200$. e Direct immunofluorescence with salt split, deposition on the dermal surface.

after the hematoxylin and eosin staining included epidermolysis bullosa acquisita (EBA) and bullous pemphigoid. Salt split immunofluorescence was performed and revealed positive immunoreactivity for IgG and C3 in a linear pattern along the floor of the blister. These findings established a diagnosis of EBA.

After her initial hospitalization, her prednisone dose was increased to $40 \mathrm{mg}$, with some improvement, and she was transitioned to azathioprine as an outpatient. Due to pancytopenia and lack of efficacy, azathioprine was discontinued. The patient had regularly been on prednisone of varying doses and had also completed trials with dapsone, multiple cycles of intravenous immunoglobulin as well as Rituxan. Despite the multiple therapies in addition to 
Butler et al.: An 81-Year-Old Woman with Recalcitrant Blisters

topical and oral steroids, the patient's disease still persists particularly in areas of friction, trauma, or contact. On her most recent admission, she received an upper endoscopy due to food impaction, and erosions with bullae were found lining her oropharynx.

\section{Discussion}

EBA is a rare mucocutaneous blistering disorder with typical onset in adulthood [1]. The mechanism behind the development of the disease is antibody production to type VII collagen at the dermal-epidermal junction resulting in disruption of the cutaneous integrity $[2,3]$. The clinical presentation of the disease is variable and attempts have been made to classify subgroups based on clinical presentation. The two main subtypes include classical EBA and inflammatory EBA.

Classical, noninflammatory EBA presents with erosions and bullae in areas of common, repeated microtrauma including the hands, feet, elbows, and knees. Lesions often heal with scarring and milia. The worst cases can result in severe scarring endpoints including fibrosis of the digits, nail loss, alopecia, and esophageal stenosis in mucosal disease [4]. Lesions of the mucosa are observed in a majority of cases and occur in both inflammatory and noninflammatory subtypes [5].

Inflammatory EBA makes up the other subtype of cases and draws its name from clinical and pathologic resemblance to other inflammatory, autoimmune disorders. In contrast to the classical type, skin fragility is not as common a feature. The most common presentations include bullous pemphigoid-like EBA, mucous membrane pemphigoid-like EBA, IgA bullous dermatosis-like EBA and Brunsting-Perry pemphigoid-like EBA [6-8].

Given the clinical and pathologic overlap with EBA and other bullous diseases, the diagnosis can be challenging. Common differential diagnoses include bullous pemphigoid, linear IgA bullous dermatosis, porphyria cutanea tarda, bullous systemic lupus erythematosus, or recessive dystrophic epidermolysis bullosa. A thorough history in addition to histologic evaluation is needed to help establish a diagnosis. Commonly, two biopsies are needed of lesional (for hematoxylin and eosin staining) and perilesional (for direct immunofluorescence) skin to confirm the diagnosis and direct immunofluorescence is the gold standard [9].

Histological evaluation varies based on the age of the lesion as well as the clinical subtype. Variation can include vacuolar changes with papillary edema in early lesions, frank blisters in developed lesions, and dermal fibrosis in older lesions $[9,10]$. Moreover, the clinical subtypes can be differentiated on pathologic evaluation. The classical, noninflammatory type, as its name would suggest, has a sparse lymphocytic infiltrate while inflammatory types involve significant perivascular, perifollicular, and intradermal inflammation. Cell types also vary and include neutrophils, lymphocytes, and eosinophils as well as variants including mixed infiltrates [10].

Direct immunofluorescence remains the gold standard test; however, it is key to remember the need for membrane zone splitting as part of this evaluation. The membrane split is essential to this diagnosis as standard direct immunofluorescence shows deposition of IgG at the dermal-epidermal junction: a finding which does not provide definitive evidence of EBA as this is shared by multiple other skin diseases [11,12]. Membrane splitting is a technique by which the lamina lucida is split by the use of sodium chloride or ethylenediaminetetraacetic acid. This subsequently allows for visualization of immunoreactants on respective sides of the lamina lucida. In the case of EBA, collagen VII is found on the dermal side and thus immunoreactants will be visualized on the "floor" of the split. This is in contrast to bullous pemphigoid where the target antigen is most commonly on the epidermal side of the split and these reactants are seen on the "roof." While serving as the gold standard, studies do show 
that up to $1 / 3$ of patients have a negative membrane split evaluation presumably due to a low level of type VII collagen $[13,14]$.

It is important to establish a diagnosis as EBA is associated with a variety of systemic diseases, and the onset of skin disease should raise clinical concern about these associations. Diseases such as inflammatory bowel disease, systemic lupus, amyloidosis, thyroiditis, rheumatoid arthritis, thymomas, chronic lymphocytic leukemia, and diabetes are noted $[15,16]$. There is no standard workup for these associated conditions; however, clinical teams should be aware of them and pursue workup on an individual basis.

\section{Statement of Ethics}

The study was prepared in compliance with all ethical and confidentiality guidelines and principles.

\section{Disclosure Statement}

The authors have no conflicts of interest to disclose.

\section{References}

1 Iranzo P, Herrero-Gonzalez JE, Mascaro-Galy JM, Suarez-Fernandez R, Espana A: Epidermolysis bullosa acquisita: a retrospective analysis of 12 patients evaluated in four tertiary hospitals in Spain. Br J Dermatol 2014;171:1022-1030.

2 Burgeson RE: Type VII collagen, anchoring fibrils, and epidermolysis bullosa. J Invest Dermatol 1993;101: 252-255.

-3 Sakai LY, Keene DR, Morris NP, Burgeson RE: Type VII collagen is a major structural component of anchoring fibrils. J Cell Biol 1986;103:1577-1586.

-4 Shipman AR, Agero AL, Cook I, et al: Epidermolysis bullosa acquisita requiring multiple oesophageal dilatations. Clin Exp Dermatol 2008;33:787-789.

5 Luke MC, Darling TN, Hsu R, et al: Mucosal morbidity in patients with epidermolysis bullosa acquisita. Arch Dermatol 1999;135:954-959.

-6 Gammon WR, Briggaman RA, Wheeler CE Jr: Epidermolysis bullosa acquisita presenting as an inflammatory bullous disease. J Am Acad Dermatol 1982;7:382-387.

7 Hashimoto T, Ishiko A, Shimizu H, et al: A case of linear IgA bullous dermatosis with IgA anti-type VII collagen autoantibodies. Br J Dermatol 1996;134:336-339.

8 Kurzhals G, Stolz W, Meurer M, Kunze J, Braun-Falco 0, Krieg T: Acquired epidermolysis bullosa with the clinical feature of Brunsting-Perry cicatricial bullous pemphigoid. Arch Dermatol 1991;127:391-395.

9 Ackerman AB CN, Sanchez J, et al: Histologic Diagnosis of Inflammatory Skin Diseases: An Algorithmic Method Based on Pattern Analysis, ed 2. Baltimore, Williams \& Williams, 1997.

10 Weedon D: The vesicobullous reaction pattern; in Weedon D (ed): Skin Pathology ed 3. New York, Elsevier, 2010

11 Yaoita H, Briggaman RA, Lawley TJ, Provost TT, Katz SI: Epidermolysis bullosa acquisita: ultrastructural and immunological studies. J Invest Dermatol 1981;76:288-292.

12 Smoller BR, Woodley DT: Differences in direct immunofluorescence staining patterns in epidermolysis bullosa acquisita and bullous pemphigoid. J Am Acad Dermatol 1992;27(5 Pt 1):674-678.

13 Wozniak K, Kazama T, Kowalewski C: A practical technique for differentiation of subepidermal bullous diseases: localization of in vivo-bound IgG by laser scanning confocal microscopy. Arch Dermatol 2003;139: $1007-1011$.

14 Hoang-Xuan T, Robin H, Heller M, Caux F, Prost C: Epidermolysis bullosa acquisita diagnosed by direct immunoelectron microscopy of the conjunctiva. Ophthalmology 1997;104:1414-1420.

15 Roenigk HH Jr, Ryan JG, Bergfeld WF: Epidermolysis bullosa acquisita. Report of three cases and review of all published cases. Arch Dermatol 1971;103:1-10.

16 Chen M, Kim GH, Prakash L, Woodley DT: Epidermolysis bullosa acquisita: autoimmunity to anchoring fibril collagen. Autoimmunity 2012;45:91-101. 